\title{
ARTICLE \\ The orthopaedic management of myelomeningocele
}

\author{
A Horn, ${ }^{1}$ MB ChB; S Dix-Peek, ${ }^{2}$ MB BCh, FCS SA (Orth), MMed; S Mears, ${ }^{2}$ MB ChB, FCS SA (Orth); \\ E B Hoffman, ${ }^{2} \mathrm{MB}$ ChB, FCS SA (Orth) \\ ${ }^{1}$ Department of Orthopaedics, Faculty of Health Sciences, University of Cape Town, South Africa \\ ${ }^{2}$ Paediatric Orthopaedics, Red Cross War Memorial Children's Hospital and University of Cape Town, South Africa
}

Corresponding author: S Dix-Peek (sdixpeek@gmail.com)

\begin{abstract}
Despite improvement in antenatal care and screening, myelomeningocele remains the most common congenital birth defect, with a reported incidence of 1 - 2.5/1 000 patients in the Western Cape, South Africa. The multidisciplinary team involved in the Spinal Defects Clinic at Red Cross War Memorial Children's Hospital, Cape Town, South Africa consists of neurosurgeons, urologists, orthopaedic surgeons, stomatherapists and orthotists. Orthopaedic surgeons have a protean involvement in the management of myelomeningocele.
\end{abstract}

S Afr Med J 2014;104(4):314. DOI:10.7196/SAMJ.8160

\section{Goals of treatment and} walking potential

Myelomeningocele is the most common congenital birth defect, with a reported incidence of $1-2.5 / 1000$ patients in the Western Cape, South Africa. ${ }^{[1]}$ A holistic appoach to a patient with spina bifida involves that the treating orthopaedic surgeon realises that mobility is only one of many treatment priorities. These patients' needs, in order of importance, are a stable self-image, an adult sexual role, independent communication, daily living activities, a career and, lastly, mobility. ${ }^{[2]}$ Orthopaedic interventions should therefore be simple and tailored to the patient's walking ability, based on neurological level. As these patients age, they tend to gain weight, thereby increasing the work of ambulation to the point where wheelchair mobility becomes preferable to walking.

The ambulatory potential of the myelomeningocele patient according to the level of neurological involvement is summarised in Table $1 .{ }^{[3]}$ Neurological level, defined as the lowest level with at least MRC grade $3 / 5$ power, is the most important determinant of walking potential.

Orthopaedic problems encountered in each neurological level are summarised in Table 2. The different deformities, based on topography, are discussed with regard to presentation and management.

\section{Spinal deformity}

Spinal deformity occurs almost exclusively in paraplegic groups of patients. Scoliosis is a result of the weak or paralysed paraspinal musculature and usually develops by the age of 10 years. The resulting pelvic obliquity leads to loss of sitting balance and necessitates the use of the patient's arms for support, thereby reducing function. Asymmetrical buttock pressure distribution and the absence of sensation may result in ischial pressure sores. Surgical scoliosis correction is reserved for curves exceeding $50^{\circ}$

and involves instrumented fusion from $\mathrm{T} 2$ to the pelvis (Fig. 1). ${ }^{[4]}$ This corrects sitting balance, but decreases lumbosacral mobility. It may also cause difficulty in wheelchair transfer and an increased propensity to develop trophic ulcers. ${ }^{[5]}$

Table 1. Walking potential as per neurological level ${ }^{[3]}$

\begin{tabular}{llll}
\hline Level & Function & Muscle & Walking potential \\
\hline Thoracic & Paraplegic & & Non-walker \\
L1 - L2 & Paraplegic, hip flexion & Psoas & Non-walker \\
L3 & Knee extension & Quadriceps & $\begin{array}{l}33 \% \text { walk at } 4-5 \text { years with } \\
\text { LF }\end{array}$ \\
& Knee extension & Quadriceps & 100\% walk at 3 - 4 years \\
& Knee flexion & Medial hamstrings & with AFOs and crutches \\
L5 & Ankle dorsiflexion & Tibialis anterior & $100 \%$ walk at 2 - 3 years \\
& & & with AFOs \\
S1 & Ankle plantar flexion & Gastroc soleus & $100 \%$ walk at $1-2$ years \\
AFOs = ankle-foot arthroses. & &
\end{tabular}

Table 2. Orthopaedic problems for each neurological level

\begin{tabular}{|c|c|c|}
\hline Level & $\%$ & Deformity \\
\hline Thoracic (paraplegic) & 23 & $\begin{array}{l}\text { Scoliosis } \\
\text { Kyphosis } \\
\text { Equinus }\end{array}$ \\
\hline Mid-lumbar (L3,4) & 30 & $\begin{array}{l}\text { Hip dislocation } \\
\text { Internal and external tibial torsion } \\
\text { Clubfoot } \\
\text { Congenital vertical talus } \\
\text { Ankle and hindfoot valgus }\end{array}$ \\
\hline Low lumbar/calcaneus (L5) & 17 & $\begin{array}{l}\text { Calcaneus } \\
\text { Ankle valgus } \\
\text { Hindfoot valgus }\end{array}$ \\
\hline Sacral/'normal' (S1) & 30 & Cavovarus foot \\
\hline
\end{tabular}


Kyphotic deformity develops at the site of the spinal dysraphism as a result of the lateralised erector spinae muscles becoming perverted flexors of the spine. The severe kyphosis leads to loss of sitting balance, early satiety and reduced pulmonary capacity, but the main concern is recurrent ulceration over the bony vertebral prominence. Development of this deformity can be prevented by excising the involved vertebrae during neurosurgical intervention shortly after birth. In the case of delayed presentation and a fixed, rigid kyphotic deformity, kyphectomy and posterior fusion can be performed, thereby lifting the chest off the abdomen and increasing spinal height (Fig. 2).

\section{Hip}

Muscle imbalance (absent abductors and/or extensors) in the myelomeningocele patient results in subluxation or dislocation of the hip, which is pushed laterally and superiorly. This leads to secondary bony deformation of the femoral neck and acetabulum (Fig. 3).

Walking ability is not affected by dislocation of the hip, but rather by the neurological level of involvement. ${ }^{[6]}$ Only $33 \%$ of L3 patients can walk, and then usually with ankle-foot orthoses (AFOs) and crutches, but all L4 patients are ambulatory. ${ }^{[7]}$ The better walking potential of $\mathrm{L} 4$ patients is due to the presence of medial hamstrings that account for a third of hip extension strength. In myelomeningocele patients, unlike in those with cerebral palsy, an unstable hip does not result in painful arthritis. It is rarely necessary to treat an unstable hip surgically, the exception being certain ambulatory patients with asymmetrical involvement.

\section{Knee}

The knee rarely requires surgery in ambulatory patients. Hyperextension deformities respond well to serial plasters, particularly in patients $<1$ year of age. If persistent, quadriceps and capsular release procedures can be performed.

Flexion deformities $<20^{\circ}$ can be treated with serial plasters and deformities up to $40^{\circ}$ are treated with an open capsular release, with or without hamstring tenotomies. If the deformity is $>40^{\circ}$, gradual correction with an external fixator is the treatment of choice.

\section{Tibial torsion}

Persistent internal torsion in patients $>5$ years of age with a mid-lumbar myelomeningocele is treated with a supramalleolar de-rotation osteotomy and T-plate fixation (Fig. 4). External tibial torsion is seen in older children. It can aggravate existing knee and ankle valgus and is treated similarly.

\section{Foot}

The aim of treatment of foot deformities is to obtain a plantigrade, mobile and braceable foot, and to avoid trophic ulceration that leads to significant morbidity that may culminate in amputation. An important study by Maynard et al. ${ }^{[8]}$ found that $94 \%$ of feet of patients with myelomeningocele require surgery. Postoperatively, the incidence of trophic ulceration in flexible plantigrade feet was $0 \%$, in flexible nonplantigrade feet $25 \%$, in rigid plantigrade feet $36 \%$, and in rigid non-plantigrade feet $100 \%$. It is therefore imperative to obtain a plantigrade foot and to avoid operations that create stiffness.

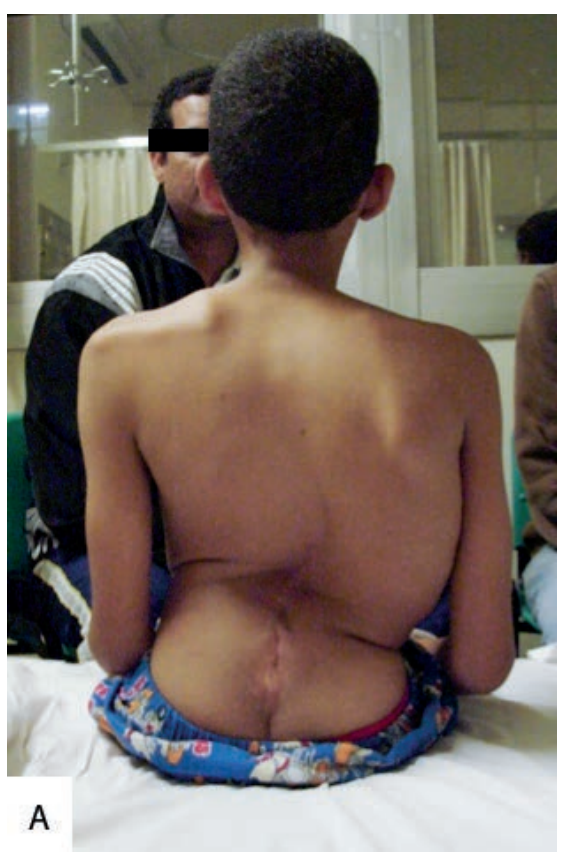

\section{Equinus}

Equinus deformity often occurs in paraplegic patients and those with a midlumbar myelomeningocele. Our policy is to treat equinus deformity at 1 year of age to facilitate transfer in paraplegic patients and ambulation in mid-lumbar patients. An Achilles tenotomy, or if required a posterior release (Achilles tenotomy, capsule and ligament release), results in a plantigrade, mobile foot that prevents point loading and pressure sores.

\section{Clubfoot}

Clubfoot occurs almost exclusively in midlumbar patients. Early treatment with the

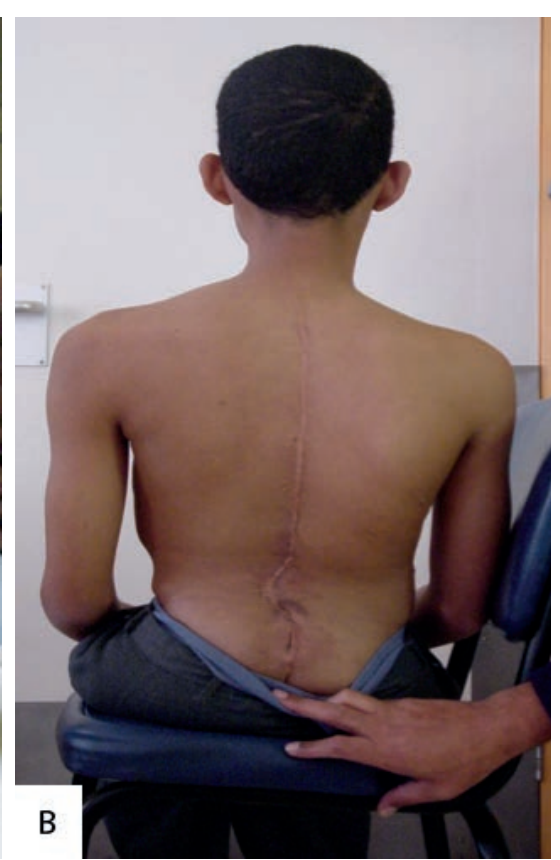

Fig. 1. (A) Severe scoliosis in a patient with high lumbar myelomeningocele. (B) Significant improvement in sitting balance following instrumented fusion.
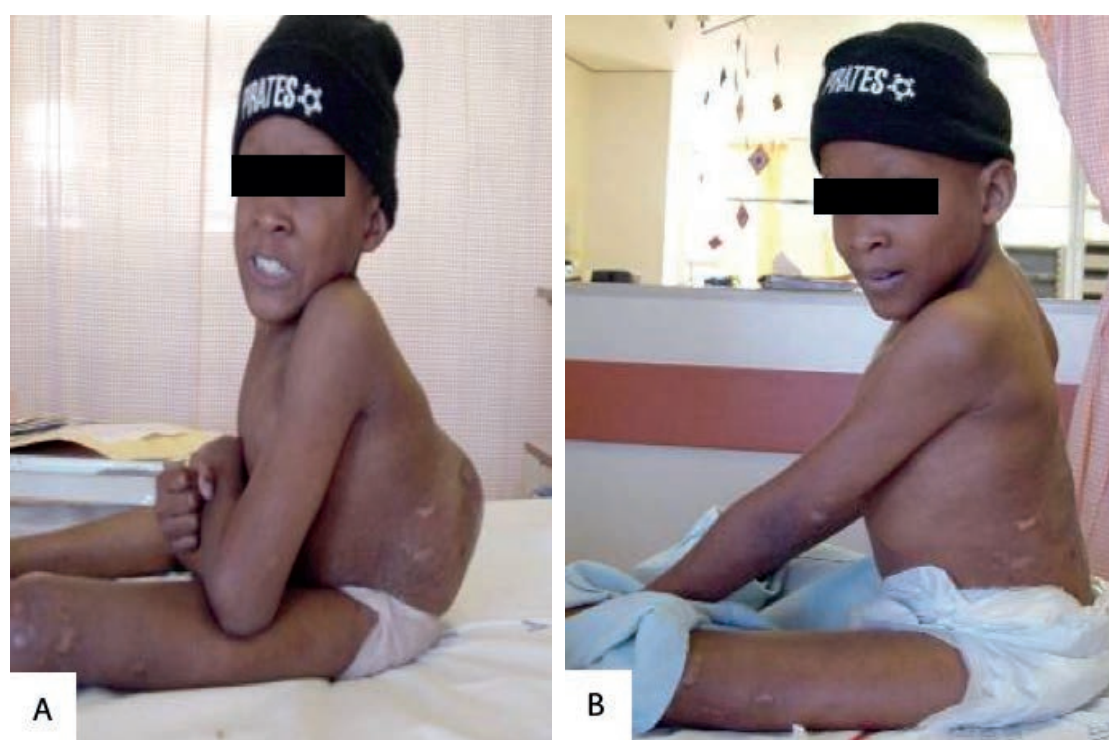

Fig. 2. (A) Patient with marked kyphotic deformity. (B) Patient after kyphectomy and posterior fusion. 
Ponseti method, which consists of weekly serial casting followed by bracing, does not cause subtalar stiffness. However, some patients' feet are very stiff and resistant to Ponseti manipulation, and a posteromedial release may be necessary. The mobility of the subtalar joint after posteromedial release in myelomeningocele patients is not predictable and therefore a plantigrade foot is imperative to avoid trophic ulceration. Some feet are very rigid and require a talectomy as a primary procedure or after a recurrence.

\section{Congenital vertical talus}

Congenital vertical talus is one of the causes of a rigid flat foot, which clinically is the reverse of a clubfoot (Fig. 5). The treatment goals are the same as for clubfoot, i.e. a flexible, plantigrade foot. Although uncommon, myelomeningocele-related congenital vertical talus comprises a third of cases in our unit. It is treated with surgical anterior and posterior release.

\section{Valgus}

A valgus foot occurs in the mid-lumbar and L5 level in myelomeningocele. In ankle valgus the callosity is under the medial malleolus and in hindfoot valgus under the talar head.

Ankle valgus is due to shortening of the fibula, with secondary lateral wedging of the distal tibial epiphysis and lateral tilt of the talus. In the immature patient, stapling of the distal medial tibial growth plate provides adequate correction (Fig. 6) ${ }^{[9]}$ In the mature patient, a varus supramalleolar osteotomy described by Wiltse $^{[10]}$ is the best option. It centralises the mechanical axis of the tibia over the talus and there is no prominent medial malleolus found after medial wedge removal.

Hindfoot valgus is due to muscle imbalance, with shortening of the Achilles tendon, and a mid-foot break. It may lead to pressure sores under the talus or navicula. Once established, it is corrected by hindfoot surgery. Historically, a subtalar fusion was the operation of choice, but this leads to subtalar stiffness. Calcaneal lengthening that does not result in a stiff subtalar joint is preferable to subtalar arthrodesis.

\section{Calcaneus}

This deformity occurs in patients with low lumbar myelomeningocele. Muscle imbalance favours ankle dorsiflexion (L5), and there is a lack of plantar flexion (S1), resulting in a calcaneus gait. This results in a weak push-off and a crouch gait, but more importantly a trophic ulcer of the insensate heel that can lead to calcaneal osteitis and eventually destruction of the calcaneus (Fig. 7). If the deformity is dynamic, transfer of the tibialis anterior tendon to the heel may be performed. ${ }^{[11]}$ If there is severe cavus with a bony deformity of the calcaneus, an osteotomy and a plantar release are required.

\section{Cavovarus}

Cavovarus occurs in S1 level myelomeningocele patients owing to an imbalance between the intrinsic and extrinsic foot muscles. There is motor function to peroneus longus, a plantarflexed first ray, but less power to the intrinsic
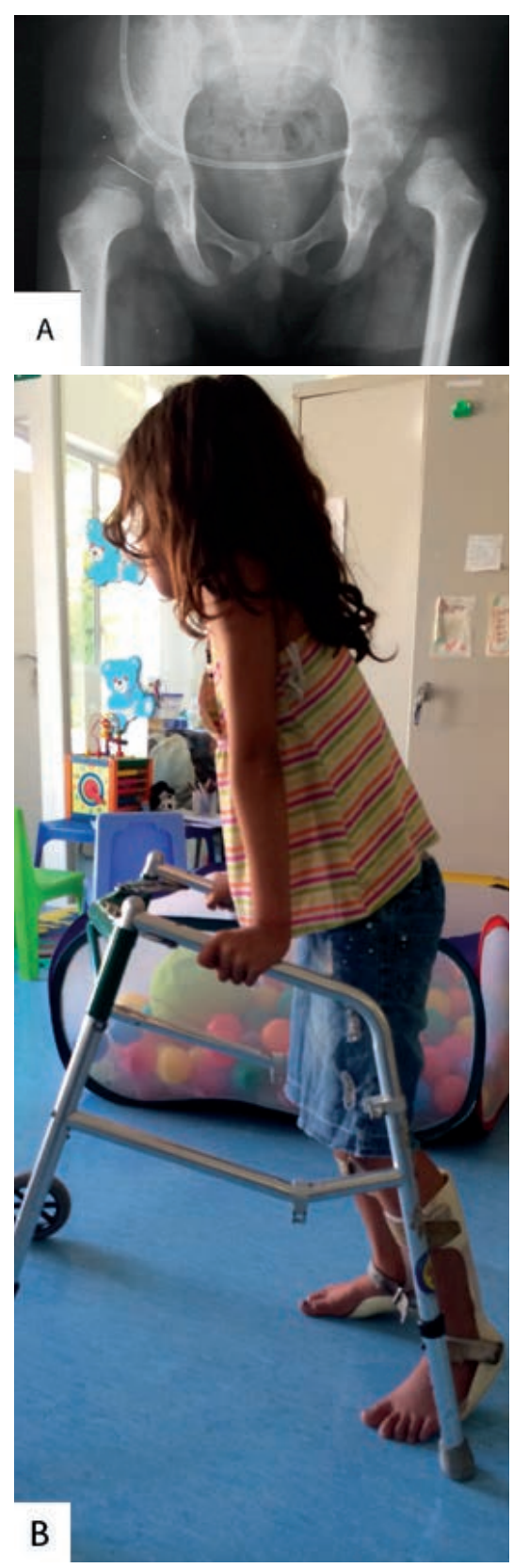

Fig. 3. (A) L4 myelomeningocele: underdeveloped femoral heads, dysplastic acetabuli, dislocated left hip and subluxated right hip. (B) Patient with mid-lumbar L4 level walking with anklefoot arthroses and crutches in spite of bilateral dislocated hips.

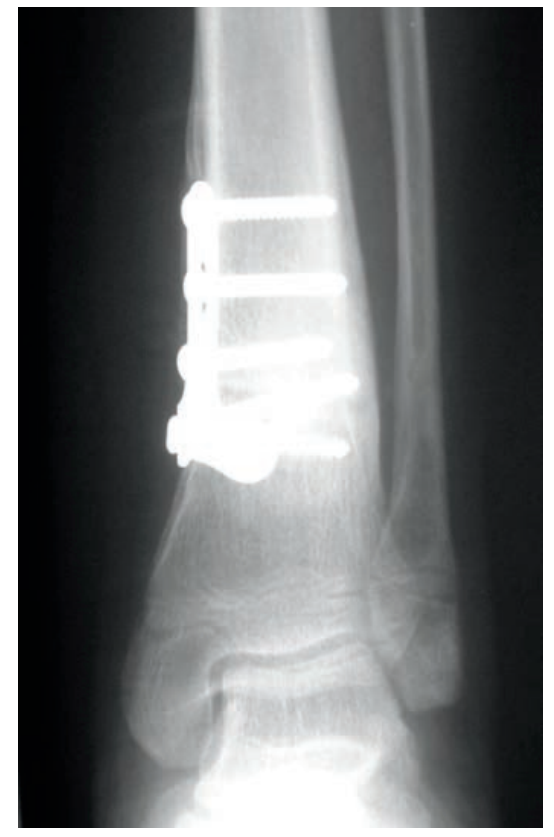

Fig. 4. T-plate fixation after de-rotation tibial osteotomy.

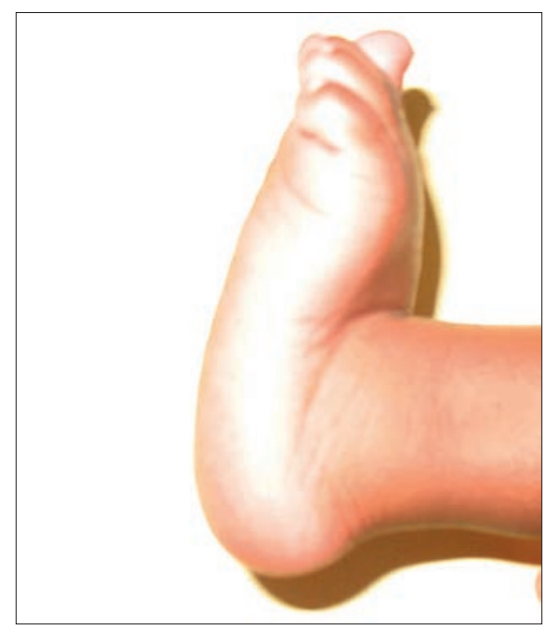

Fig. 5. Rocker bottom deformity in congenital vertical talus.

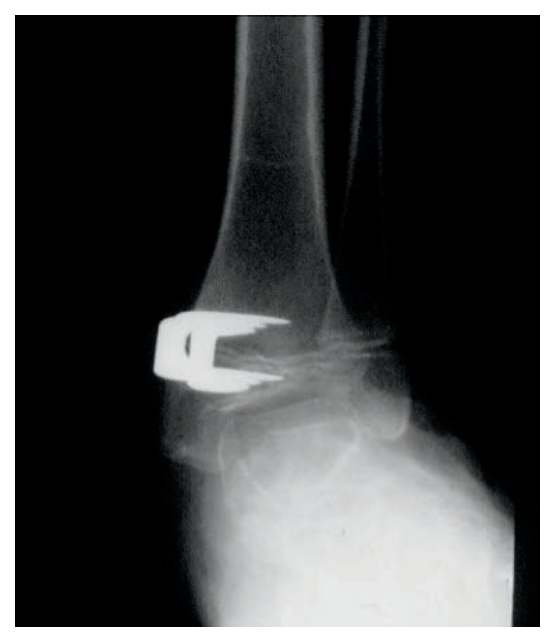

Fig. 6. Stapling of the medial distal tibial physis to correct valgus deformity. 


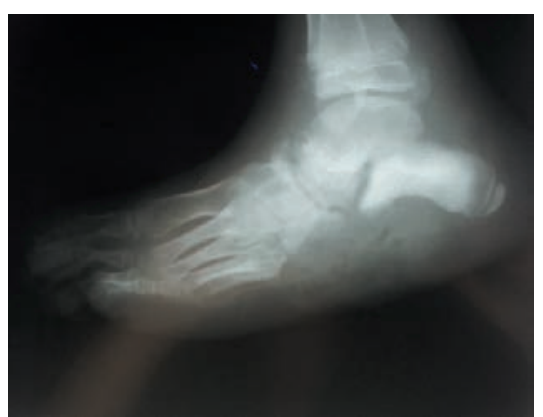

Fig. 7. Calcaneal and mid-foot osteitis in an L5 myelomeningocele.

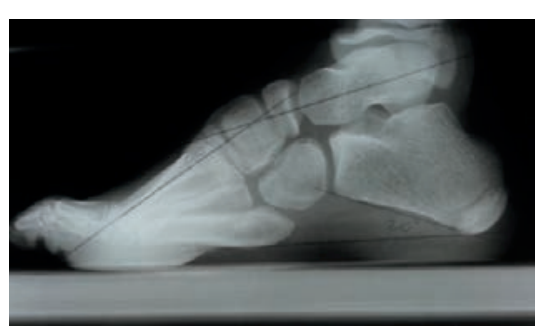

Fig. 8. Typical radiographic appearance of a cavus deformity.

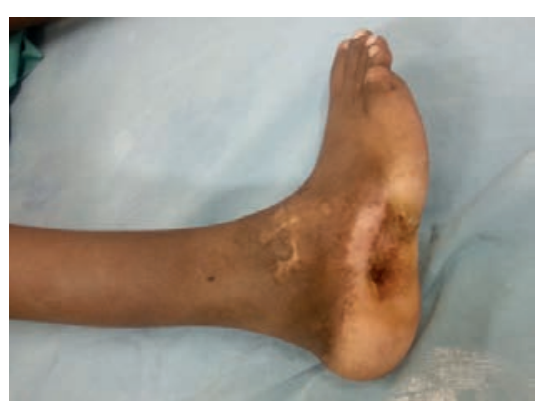

Fig. 9. Trophic ulcer on the lateral border of the foot secondary to hindfoot varus in a patient with L5 myelomeningocele. muscles that extend the toes. The eventual plantar-flexed first ray thereafter drives both cavus, resulting in hindfoot varus (Fig. 8). Treatment is dictated by the mobility of the subtalar joint. If mobile, surgery is via softtissue release and tendon transfer - usually plantar release and peroneus longus to brevis tendon transfer. If stiff, then soft-tissue and bony procedures, such as a lateral closing wedge osteotomy of the calcaneus, are required.

\section{Trophic ulcers}

Trophic ulcers of the foot are due to a stiff, non-plantigrade foot or a neglected injury in an insensate foot. The surgeon should always endeavour to obtain a plantigrade, mobile foot. In a neglected injury, the normal subcutaneous fat pad of the sole of the foot has been destroyed, and if the ulcer heals by secondary intent with scar tissue, it invariably breaks down again. A trophic ulcer is treated by reconstituting a normal skin and fat pad by means of a medially based rotation flap. This should be preceded by correction of a non-plantigrade foot and removal of bony prominences under the ulcer. If the ulcer is on the heel, the posterior calcaneus can be displaced superiorly with surgery (Fig. 9).

\section{Charcot arthropathy and fractures}

Both Charcot arthropathy and fractures can present with an exaggerated clinical response of swelling, warmth and erythema. It is usually incorrectly diagnosed as cellulitis, septic arthritis or osteitis. In Charcot arthropathy the initial radiographs are normal and the joint should be protected (first in a cast for 6 weeks and then with an orthosis) to prevent further joint destruction. Fractures develop exuberant callus. In diaphyseal and metaphyseal fractures there is usually a history of trauma, requiring appropriate immobilisation. Physeal fractures are due to repetitive stress fractures in a poorly sensate limb and, similar to Charcot arthropathy, require long-term orthotic splinting after the initial cast immobilisation. Physeal bar formation with growth arrest can occur.

\section{References}

1. Buccimazza SS, Molteno CD, Dunne TT, Viljoen DI. Prevalence of neural tube defects in Cape Town, South Africa. Teratology 1994;50(3):194-199. [http://dx.doi.org/10.1002/ tera.1420500304]

2. Holmbeck GN, Westhoven VC, Phillips WS, et al. A multimethod, multi-informant and multidimensional perspective on psychological adjustment in preadolescents with spina bifida. J Consult Clin Psychol 2003;71(4):782-796. [http:// dx.doi.org/10.1037/0022-006X.71.4.782

3. Hoffer MM, Feiwell E, Perry R, et al. Functional ambulation in patients with myelomeningocele. J Bone Joint Surg Am 1973;55(A):137-148.

4. Widman RF, Hresko MT, Hall JE. Lumbosacral fusion in children and adolescents using the modified sacral bar technique. Clin Orthop 1999;364:85-91. [http://dx.doi.org/10.1097/00003086199907000-00012]

5. Wild A, Hack H, Kumar M, et al. Is sacral instrumentation mandatory to address pelvic obliquity in neuromuscular thoracolumbar scoliosis due to myelomeningocele? Spine 2001;26:E325-E329. [http://dx.doi.org/10.1097/00007632200107150-00019]

6. Bazih J, Gross RH. Hip surgery in the lumbar myelomeningocele patient. J Pediatr Orthop 1981:1:405-411. [http://dx.doi org/10.1097/01241398-198112000-00009]

7. Fraser RK, Hoffman EB, Sparks LT, et al. The unstable hip and mid-lumbar myelomeningocele. J Bone Joint Surg $\mathrm{Br}$ 1992;74(B):143-146.

8. Maynard MJ, Weiner LS, Burke SW. Neuropathic foot ulceration in patients with myelodysplasia. J Pediatr Orthop 1992;12:786788. [htp.//dx.doi.og/.1097/241398-199211000-00016] Burkus JK, Moore DW, Raycroft JF. Valgus deformity of the ankle in myelodysplasia patients. Correction by stapling of the medial part of the distal tibial physis. J Bone Joint Surg Am
1983;65(A):1157-1162.

10. Wiltse LL. Valgus deformity of the ankle. A sequel to acquired or congenital abnormalities of the fibula. J Bone Joint Surg Am or congenital abnorn
1972;54(A):595-606.

11. Fraser RK, Hoffman EB. Calcaneus deformity in the ambulant myelomeningocele patient. J Bone Joint Surg Br 1991;73(B):615621. 